\title{
Identification of IncRNAs associated with the pathogenesis of ankylosing spondylitis
}

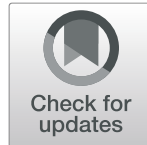

Dan Huang ${ }^{1}$, Jian Liu ${ }^{1,2^{*}}$ D, Lei Wan ${ }^{1,2}$, Yanyan Fang ${ }^{1}$, Yan Long ${ }^{1}$, Ying Zhang ${ }^{1}$ and Bingxi Bao ${ }^{1}$

\begin{abstract}
Background: Ankylosing spondylitis (AS) is a chronic autoimmune disease affecting the sacroiliac joint. To date, few studies have examined the association between long non-coding RNAs (IncRNAs) and AS pathogenesis. As such, we herein sought to characterize patterns of AS-related IncRNA expression and to evaluate the potential role played by these IncRNAs in this complex autoimmune context.

Methods: We conducted a RNA-seq analysis of peripheral blood mononuclear cell (PBMC) samples isolated from five AS patients and corresponding controls. These data were then leveraged to characterize AS-related IncRNA expression patterns. We further conducted GO and KEGG enrichment analyses of the parental genes encoding these IncRNAs, and we confirmed the validity of our RNA-seq data by assessing the expression of six IncRNAs via qRT-PCR in 15 AS and control patient samples. Pearson correlation analyses were additionally employed to examine the associations between the expression levels of these six IncRNAs and patient clinical index values.

Results: We detected 56,575 total IncRNAs in AS and control patient samples during our initial RNA-seq analysis, of which 200 and 70 were found to be up- and down-regulated (FC $>2$ or $<0.05 ; P<0.05$ ), respectively, in AS samples relative to controls. In qRT-PCR validation assays, we confirmed the significant upregulation of NONHSAT118801.2, ENST00000444046, and NONHSAT183847.1 and the significant downregulation of NONHSAT205110.1, NONHSAT105444.2, and NONHSAT051856.2 in AS patient samples. We further found the expression of NONHSAT118801.2 and NONHSAT183847.1 to be positively correlated with disease severity.
\end{abstract}

Conclusion: Overall, our findings highlight several IncRNAs that are specifically expressed in PBMCs of AS patients, indicating that they may play key functions in the pathogenesis of this autoimmune disease. Specifically, we determined that NONHSAT118801.2 and NONHSAT183847.1 may influence the occurrence and development of AS.

Keywords: Ankylosing spondylitis, IncRNAs, mRNA, RNA-seq

\footnotetext{
*Correspondence: liujianahzy@126.com

${ }^{1}$ Department of Rheumatology, First Affiliated Hospital of Anhui University of Chinese Medicine, No 117 Meishan Road, Shushan District, Hefei City, Anhui Province 230031, People's Republic of China

${ }^{2}$ Rheumatology institute of Anhui Academy Chinese Medicine, No 117 Meishan Road, Shushan District, Hefei City, Anhui Province 230031, People's Republic of China
}

(c) The Author(s). 2021 Open Access This article is licensed under a Creative Commons Attribution 4.0 International License, which permits use, sharing, adaptation, distribution and reproduction in any medium or format, as long as you give appropriate credit to the original author(s) and the source, provide a link to the Creative Commons licence, and indicate if changes were made. The images or other third party material in this article are included in the article's Creative Commons licence, unless indicated otherwise in a credit line to the material. If material is not included in the article's Creative Commons licence and your intended use is not permitted by statutory regulation or exceeds the permitted use, you will need to obtain permission directly from the copyright holder. To view a copy of this licence, visit http://creativecommons.org/licenses/by/4.0/ The Creative Commons Public Domain Dedication waiver (http://creativecommons.org/publicdomain/zero/1.0/) applies to the data made available in this article, unless otherwise stated in a credit line to the data. 


\section{Background}

Ankylosing spondylitis (AS) is a chronic autoimmune inflammatory disease [1]. While AS is often asymptomatic during the early stages when it is most amenable to treatment, during the later stages of disease irreversible axial spine damage can develop, resulting in loss of mobility and debilitating pain in affected patients [2]. AS development is believed to be driven by numerous environmental and genetic factors [3], including macrophage activation status, infections with particular bacteria, and the expression of specific genes [4-6]. The exact etiology of this disease, however, remains incompletely characterized, and further in-depth analyses of the molecular basis of AS are thus warranted.

Long non-coding RNAs (lncRNAs) are RNAs $>200$ nucleotides long that largely lack coding potential, and yet are critically important in physiological and pathological contexts [7]. Indeed, cell- and disease-specific lncRNA expression patterns have been characterized to date [8]. From a functional perspective, IncRNAs can control gene and protein expression at the epigenetic, transcriptional, and post-transcriptional levels via controlling genomic imprinting, chromatin remodeling, histone modification, splicing, transcription, and cell cycle progression $[9,10]$. There is also evidence that lncRNAs are related to the incidence of autoimmune diseases including systemic lupus erythematosus [11-13], multiple sclerosis [14, 15], rheumatoid arthritis, and psoriasis [16-18]. The functional relevance of lncRNAs expressed in peripheral blood mononuclear cells (PBMCs) from AS patients, however, remain to be clarified.

Herein, we assessed AS-related IncRNA expression profiles via the RNA-seq analysis of PBMC samples from five AS patients and controls. We then used qRT-PCR to validate these RNA-seq findings. Together, our data offer a new theoretical framework for understanding the pathological basis of AS, highlighting novel diseaserelated lncRNAs for future research and therapeutic targeting efforts.

\section{Materials and methods}

\section{Sample collection}

We enrolled 15 total patients from the Department of Rheumatology at the First Affiliated Hospital of Anhui University of Chinese Medicine that had been diagnosed with AS based on the modified American College of Rheumatology (ACR) New York criteria (1984) [19]. We also enrolled 15 age- and sex-matched healthy controls. No enrolled patients had any history of prior diabetes, hepatitis, malignancies, cardiovascular disease, or other autoimmune/inflammatory diseases. All enrolled AS patients completed Bath ankylosing spondylitis diseases active index (BASDAI) [20], Bath ankylosing spondylitis functional index (BASFI) [21], and visual analog scale
(VAS) assessments. In addition, we determined key clinical and laboratory parameters for each of these patients including erythrocyte sedimentation rate (ESR), Creactive protein (CRP) levels, and immunoglobulin $\mathrm{G}$ (IgG) levels. We randomly selected five AS patients and five healthy controls for RNA sequencing analysis.

\section{PBMC preparation and total RNA extraction}

Lymphoprep (Stemcell, USA) was utilized to separate PBMCs from the blood of AS patients and controls at room temperature via density gradient centrifugation. A MiRNeasy Mini Kit (Qiagen, Germany) was then used to extract total sample RNA, after which RNA quantity was evaluated using a NanoDrop 2000 instrument (Thermo Fisher Scientific, USA), and an Agilent 2100 bioanalyzer (Agilent Technologies, USA) was utilized to establish RNA quality [22].

\section{RNA-sequencing}

A Truseq ${ }^{\circ}$ chain total RNA sample preparation kit (Illumina, USA) was used to prepare RNA libraries based upon provided instructions, after which quantification was conducted using a Qubit 2.0 fluorometer, and quality was assessed via an Agilent 2100 Bioanalyzer. CBOT was then utilized to dilute the library to $10 \mathrm{pm}$ in order to generate clusters, and an Illumina Hiseq 2500 instrument (Illumina) was utilized for sequencing. OG Biotech Inc. (AO Ji biotech, Shanghai, China) conducted all library preparation and sequencing.

\section{Data analysis}

FastQC1 (v. 0.11.3) was initially utilized for quality control (QC) assessment of RNA-seq reads, after which rRNA reads, adapter sequences, and low-quality reads were trimmed with the seqtk2 software. BWA-MEM (v2.0.4) was then used to map the trimmed reads to the human reference genome (hg38), after which CIRI was used to predict lncRNAs in these RNA-seq data, and SRPBM was used to quantify lncRNA counts therein. Next, lncRNAs that were differentially expressed between AS and control patients were detected using the EdgeR software based on the following selection criteria: $\mathrm{FC} \geq 2$ or $<0.5 ; P<0.05$. The potential functional roles of these lncRNAs were assessed via GO and KEGG enrichment analyses of the parental genes harboring these differentially expressed IncRNAs. In addition, Pearson correlation analyses were used to detect associations between lncRNA and mRNA expression profiles, with a co-expression network being constructed by incorporating interactions with a Pearson's $\mathrm{R}>0.9$ and $P<0.05$. Cytoscape was then used to visualize this lncRNAmRNA interaction network. 


\section{qRT-PCR}

To validate our RNA-seq data, we randomly selected three up-regulated (NONHSAT118801.2, ENST000004444046, and NONHSAT183847.1) and three down-regulated (NONHSAT205110.1, NONHSAT105444.2, and NONHSAT051856.2) lncRNAs from 270 differentially expressed lncRNAs via qRT-PCR in 15 AS and control samples with a Universal SYBR Green Master mix. OGBiotech Inc. synthesized all primers used for this analysis (Table 1). The $2-\Delta \Delta \mathrm{Ct}$ method was used to assess relative lncRNA expression levels.

\section{Statistical analysis}

GraphPad Prism 6.0 was used for the assessment of relative lncRNA expression and figure construction. Data are given as means \pm standard deviation (SD), and were compared via Student's t-tests. Relationships between different parameters were compared via Spearman and Pearson correlation analyses. $P<$ 0.05 was the significance threshold for this study.

\section{Results}

\section{Patient characteristics}

We detected no significant differences in age or sex when comparing the AS and control patient cohorts in the present study (Table 2).

\section{Identification of AS-related differentially expressed IncRNAs}

We next identified lncRNAs that were differentially expressed between AS and control patient PBMCs using EdgeR $(\mathrm{FC} \geq 2$ or $\leq 0.5 ; P<0.05)$. In total, we identified 270 lncRNAs that were differentially expressed between these two patient groups, of which
200 and 70 were up- and down-regulated in AS patient samples, respectively (Fig. $1 \mathrm{a}-\mathrm{c}$ ). The top 10 upand down-regulated lncRNAs identified through these analyses are compiled in Tables 3 and 4 .

\section{Pathway enrichment analyses of differentially expressed IncRNAs}

In an effort to more fully explore the potential functional roles of these differentially expressed lncRNAs, we next conducted GO and KEGG enrichment analyses of the parental genes harboring these lncRNAs using the ClusterProfler tool.

GO analyses enabled us to identify key biological processes (BPs), molecular functions (MFs), and cellular components (CCs) enriched among these lncRNAs (Fig. 2a). Significantly enriched BPs included the regulation of NF$\mathrm{\kappa B}$ nuclear import into the nucleus, responses to electrical stimuli, negative regulation of $\mathrm{I}-\mathrm{kB}$ kinase/NF- $\mathrm{kB}$ signaling, and positive regulation of transcription factor import into the nucleus, while significantly enriched CC terms included basement membrane, and significantly enriched MFs included transmembrane receptor protein tyrosine kinase activity.

In a subsequent KEGG analysis, 134 pathways were found to be significantly enriched for these lncRNAs, with the top 30 being shown in Fig. 2b. The most significantly enriched of these pathways included the TNF, PI3K-Akt, HIF-1, Rap1, Hippo, ECM-receptor interaction, and arrhythmogenic right ventricular cardiomyopathy (ARVC) signaling pathways.

Preparation of a IncRNA-mRNA co-expression network We next prepared a lncRNA-mRNA co-expression network using our RNA-seq data that incorporated

Table 1 Primers used in the present study

\begin{tabular}{lll}
\hline Gene & Primers sequences & pcr product length (bp) \\
\hline GAPDH & F: ACAACTTGGTATCGTGGAAGG & 101 \\
NONHSAT118801.2 & R: GCCATCACGCCACAGTTC & 155 \\
NONHSAT183847.1 & F: AGTCCCTGCTITAATTCTTGGGG & 133 \\
ENST00000444046 & F: GACACGGAGGCAAATCGGCTGC & 106 \\
NONHSAT205110.1 & R: GTGTGTGGGGGCAGGGGAG & 196 \\
NONHSAT105444.2 & F: CGACGGATCGGGAAAGCCAA & 196 \\
RONHSAT051856.2 & F: GCCCGTGGGAGCCTGAGAG & 81 \\
\hline
\end{tabular}


Table 2 Study population clinical characteristics

\begin{tabular}{llll}
\hline Indexes & AS & Control & $\boldsymbol{P}$ value \\
\hline Sex (M/F) & $11 / 4$ & $11 / 4$ & 1.00 \\
Age (years) & $33.60 \pm 8.24$ & $34.60 \pm 7.03$ & 0.723 \\
Disease duration (years) & $7.97 \pm 6.18$ & $\mathrm{NA}$ & $\mathrm{NA}$ \\
ESR (mm/h) & $51.27 \pm 25.50$ & $\mathrm{NA}$ & $\mathrm{NA}$ \\
CRP $(\mathrm{mg} / \mathrm{dL})$ & $55.30 \pm 35.31$ & $\mathrm{NA}$ & $\mathrm{NA}$ \\
IgG (g/L) & $13.34 \pm 3.38$ & $\mathrm{NA}$ & $\mathrm{NA}$ \\
BASDAl (score) & $6.55 \pm 0.48$ & $\mathrm{NA}$ & $\mathrm{NA}$ \\
BASFI (score) & $6.33 \pm 0.56$ & $\mathrm{NA}$ & $\mathrm{NA}$ \\
VAS (score) & $6.1 \pm 1.09$ & $\mathrm{NA}$ & $\mathrm{NA}$ \\
\hline
\end{tabular}

pairs of lncRNAs and mRNAs that yielded Pearson correlation coefficient values $\geq 0.9$, with Cytoscape being used to visualize this network. In total, 948 differentially expressed mRNA were obtained, including 628 upregulated expressed and 320 downregulated expressed. Co-expression networks generated using control and AS patient samples different substantially with respect to the number of incorporated nodes and connections (Fig. 3).

\section{RNA-seq result validation}

We next validated our RNA-seq data by assessing the expression of 6 randomly selected differentially expressed lncRNAs in PBMC samples from 15 AS and 15 control patients via qRT-PCR. This analysis enabled us to confirm that NONHSAT118801.2, ENST00000444046, and NONHSAT183847.1 were significantly upregulated in AS patient PMBCs, whereas NONHSAT205110.1, NONHSAT205110.1, and NONHSAT051856.2 were significantly downregulated in these samples (Fig. 4). The qRT-PCR results were consistent with the RNA-seq results.

\section{Correlations between IncRNA expression patterns and AS patient clinical findings}

Lastly, we evaluated the association between the expression of the identified differentially expressed lncRNAs in AS patients and patient disease status. Specifically, we conducted Pearson correlation analyses comparing the expression of the six validated lncRNAs (NONHSAT118801.2, ENST00000444046, NONHSAT183847.1, NONHSAT205110.1, NONHSAT205110.1, and NONHSAT051856.2) and AS clinical features (ESR, hs-CRP, IgG, BASDAI, BASFAI, and VAS). We determined that NONHSAT118801.2

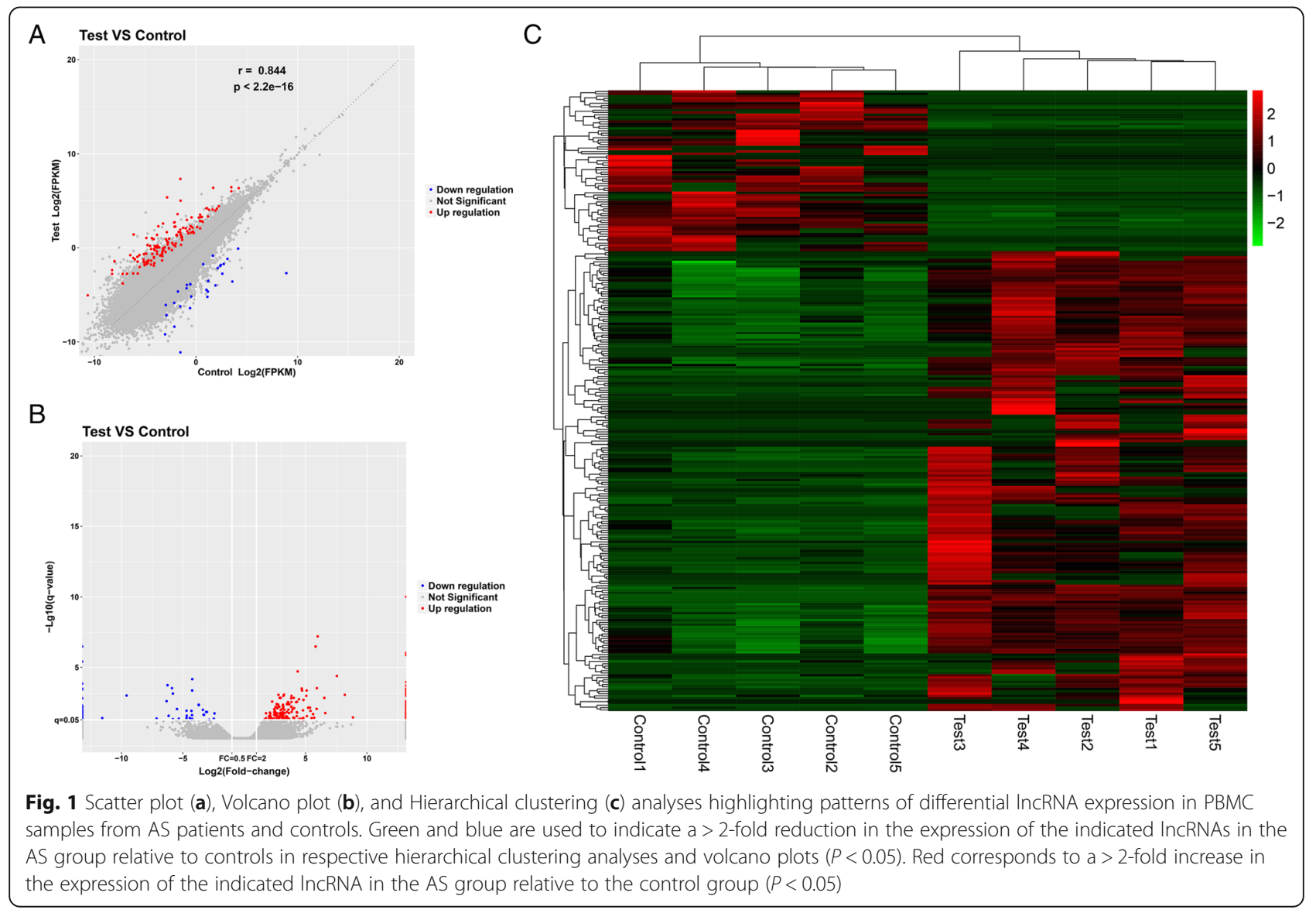


Table 3 The top 10 known differentially expressed IncRNAs that were upregulated in AS patient PMBCs relative to control samples

\begin{tabular}{llllcc}
\hline IncRNAs & locus & length & log2FC & P value & Style \\
\hline MSTRG.15374.10 & $14: 49564494-49,586,878$ & 1143 & 8.86469086799321 & $1.13 \mathrm{E}-04$ & Up \\
ENST00000592882 & $19: 13153096-13,154,193$ & 756 & 8.20263648791897 & $4.97 \mathrm{E}-07$ & Up \\
ENST00000520447 & $3: 9388883-9,397,495$ & 1809 & 7.55311232709341 & $6.47 \mathrm{E}-09$ \\
NONHSAT242907.1 & $2: 207176461-207,529,981$ & 14,966 & 6.60740194542888 & $4.01 \mathrm{E}-05$ & Up \\
NONHSAT206983.1 & $6: 26204366-26,204,807$ & 442 & 6.53317269333968 & $1.08 \mathrm{E}-06$ & Up \\
MSTRG.38467.6 & $3: 193667058-193,683,114$ & 911 & 5.99499453964437 & $2.22 \mathrm{E}-12$ & Up \\
NONHSAT243832.1 & $20: 40322988-40,323,673$ & 686 & 5.9247373389848 & $8.16 \mathrm{E}-08$ & Up \\
NONHSAT226007.1 & $1: 223992760-224,015,005$ & 15,684 & 5.90517001896817 & $1.30 \mathrm{E}-05$ & Up \\
ENST00000574306 & $17: 1711511-1,716,251$ & 2626 & 5.8167471830444 & $2.33 \mathrm{E}-11$ & Up \\
NONHSAT233477.1 & $13: 109765892-109,767,567$ & 1529 & 5.70399695982728 & $1.21 \mathrm{E}-04$ & Up \\
\hline
\end{tabular}

expression levels were positively correlated with ESR, BASDAI, and BASFAI levels in AS patients (Fig. 5ac), while NONHSAT183847.1 levels were positively associated with ESR, BASDAI, and CRP (Fig. 5d-f).

\section{Discussion}

While structurally similar to mRNAs, lncRNAs generally lack the ability to encode peptides. Although they were originally considered to be irrelevant and a form of transcriptional noise, lncRNAs are now recognized to be key regulators of gene and protein expression at the epigenetic, transcriptional, and post-transcriptional levels. These lncRNAs are thus key determinants of metabolic activity, development, evolution, and disease pathophysiology [22].

A previous microarray study identified five lncRNAs, NDRG1-AS6, CSNK1D-AS8, CD46-AS9, SMYD5-AS2, and NR_045553 that are involved in hip joint ligament tissues in patients with AS [23]. In addition, MSTRG.8559 and LINC00987 were identified as two hub differentially expressed lncRNAs in AS patients [24]. In one recent study, peripheral blood lncRNA-AK001085 levels were found to be reduced in AS patients and to be negatively correlated with CRP and ESR levels in these individuals, suggesting a potential protective role for this lncRNA in this pathological context [25]. Although the role of LncRNAs in the pathogenesis of AS has been preliminarily understood, the regulatory mechanism of most LncRNA in AS remains unclear due to its large system, complex mechanism and diverse functions. Our data provide novel insights into lncRNA expression profiles in AS patient PBMCs, as we were able to identify 270 total differentially expressed lncRNAs, including 200 and 70 that were up- and down-regulated in AS patient samples, respectively. We validated the differential expression of three up- and down-

Table 4 The top 10 known differentially expressed IncRNAs that were downregulated in AS patient PMBCs relative to control samples

\begin{tabular}{lllllr}
\hline IncRNAs & locus & length & log2FC & $P$ value & Style \\
\hline NONHSAT169063.1 & $14: 22769653-22,799,551$ & 5272 & -2.42547013179437 & $4.36 \mathrm{E}-05$ & Down \\
NONHSAT213394.1 & $7: 38239579-38,249,550$ & 1035 & -2.47555403789066 & $1.74 \mathrm{E}-04$ & Down \\
NONHSAT247151.1 & $3: 195994009-195,997,627$ & 3619 & -2.8536961853987 & $2.31 \mathrm{E}-04$ & Down \\
NONHSAT123640.2 & $7: 139430287-139,433,451$ & 3165 & -3.03849037973017 & $2.85 \mathrm{E}-05$ & Down \\
NONHSAT236815.1 & $16: 81292454-81,314,778$ & 22,325 & -3.10565434683757 & $2.80 \mathrm{E}-05$ & Down \\
NONHSAT051856.2 & $16: 717619-719,654$ & 2036 & -3.279800451942 & Down \\
NONHSAT248657.1 & $4: 141296872-141,332,617$ & 18,512 & -3.31012780603255 & $5.80 \mathrm{E}-05$ & Down \\
NONHSAT229511.1 & $10: 130061449-130,110,821$ & 10,032 & -3.37040958330436 & $1.70 \mathrm{E}-05$ & Down \\
NONHSAT230554.1 & $11: 66472049-66,480,241$ & 8193 & -3.70572335168321 & $5.12 \mathrm{E}-06$ & Down \\
NONHSAT153273.1 & $1: 244306789-244,309,614$ & 2826 & -4.23561043031746 & $1.21 \mathrm{E}-08$ & Down \\
\hline
\end{tabular}



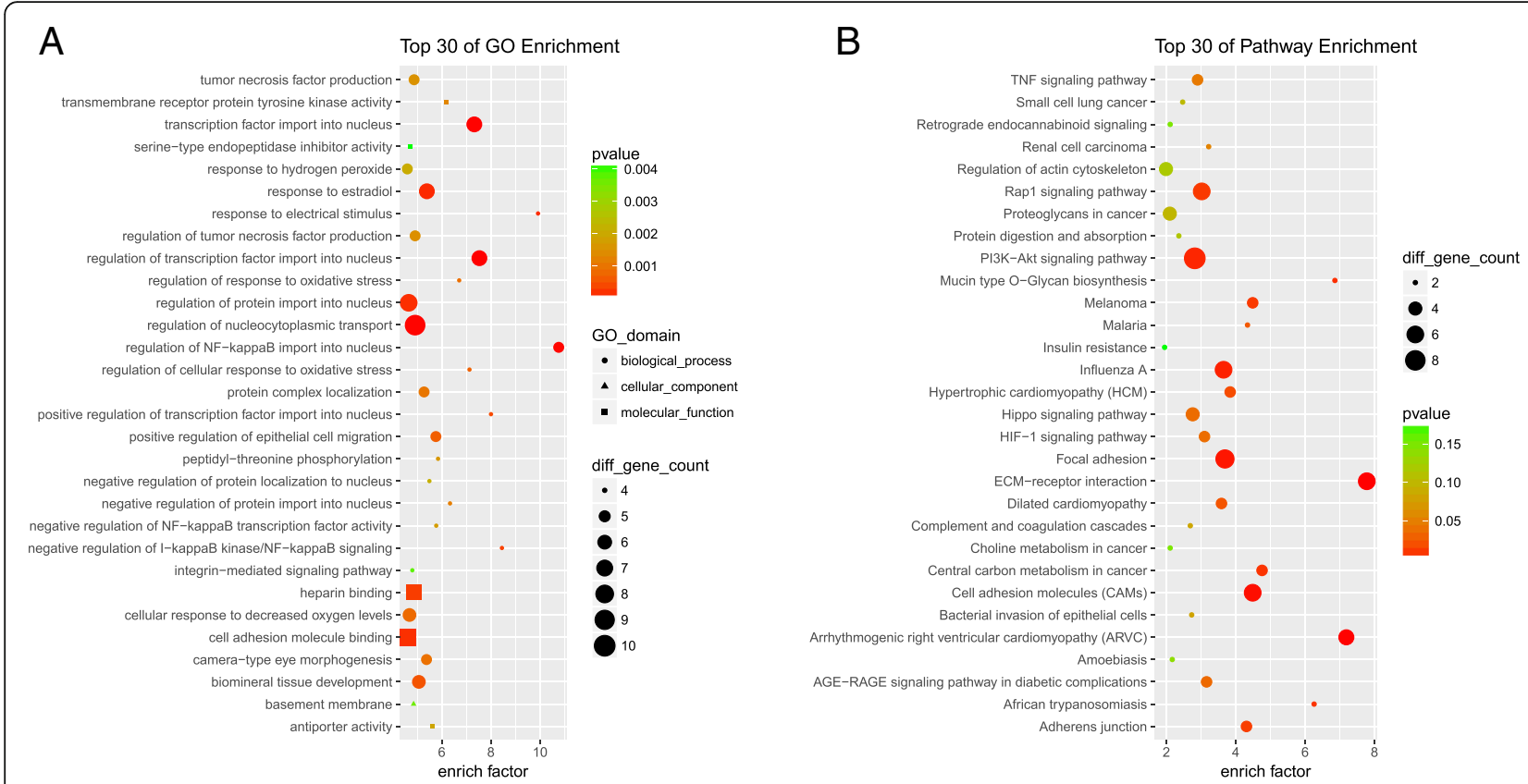

Fig. 2 The results of GO and KEGG pathway enrichment analyses of parental genes harboring AS-related differentially expressed IncRNAs. a The top 30 enriched GO enrichment terms. $\mathbf{b}$ The top 30 classes of KEGG pathway enrichment terms

regulated lncRNAs via qRT-PCR to confirm the reliability of our RNA-seq data, The qRT-PCR results were consistent with the RNA-seq resultsand selected these IncRNAs for further analysis. Together, our data have the potential to serve as a framework for future studies of the role of these lncRNAs in the context of AS disease processes.

Our GO analysis results indicate potential roles for these differentially expressed lncRNAs as important regulators of NF- $\mathrm{kB}$, which is a key transcription factor and regulator of inflammatory and oxidative stress-related processes in cells. These lncRNAs were also associated with signaling pathways including the TNF, PI3K-Akt, Rap1, Hippo, and HIF-1 pathways, which are primarily involved in inflammatory immune regulation and osteogenic differentiation. In some prior studies, the PI3K-Akt signaling pathway has been suggested to promote fibroblastic ossification and inflammation in the context of AS [26]. Some of the identified pathways, including the Rap1, Hippo, and HIF-1 pathways, have not previously been studied in the context of AS, and thus warrant future investigation.

To confirm our RNA-seq findings, we utilized a qRT-PCR approach to validate the differential expression of three up-regulated lncRNAs (NONHSAT118801.2, ENST00000444046, NONHSAT1838
47.1) and three down-regulated IncRNAs (NONHSAT205110.1, NONHSAT205110.1, NONHSAT051 856.2) in 15 AS patient samples relative to 15 control samples. This approach confirmed that all six of these lncRNAs were differentially expressed in the expected directions (all $P<0.01$ ), thereby reaffirming the reliability of our RNA-seq data. We also assessed correlations between the expression levels of these lncRNAs and AS patient disease indices, leading us to determine that NONHSAT118801.2 expression was positively correlated with ESR, BASDAI, and BASFAI levels in AS (Fig. 5a-c), while NONHSAT183847.1 expression was positively correlated with ESR, BASDAI, and CRP. These data suggest that NONHSAT118801.2 and NONHSAT183847.1 may be key regulators of the pathogenesis of AS, although additional research will be necessary in order to understand the mechanisms whereby these candidate lncRNAs impact disease progression.

Together, our data offer a new mechanistic basis for understanding the pathogenesis of AS. However, we recognized that the present study has some limitations. First, the sample size was small, necessitating the collection of a larger sample from different regions and involving subjects belonging to different races. Second, we did not assess the ability of the markers to effectively differentiate AS from other 


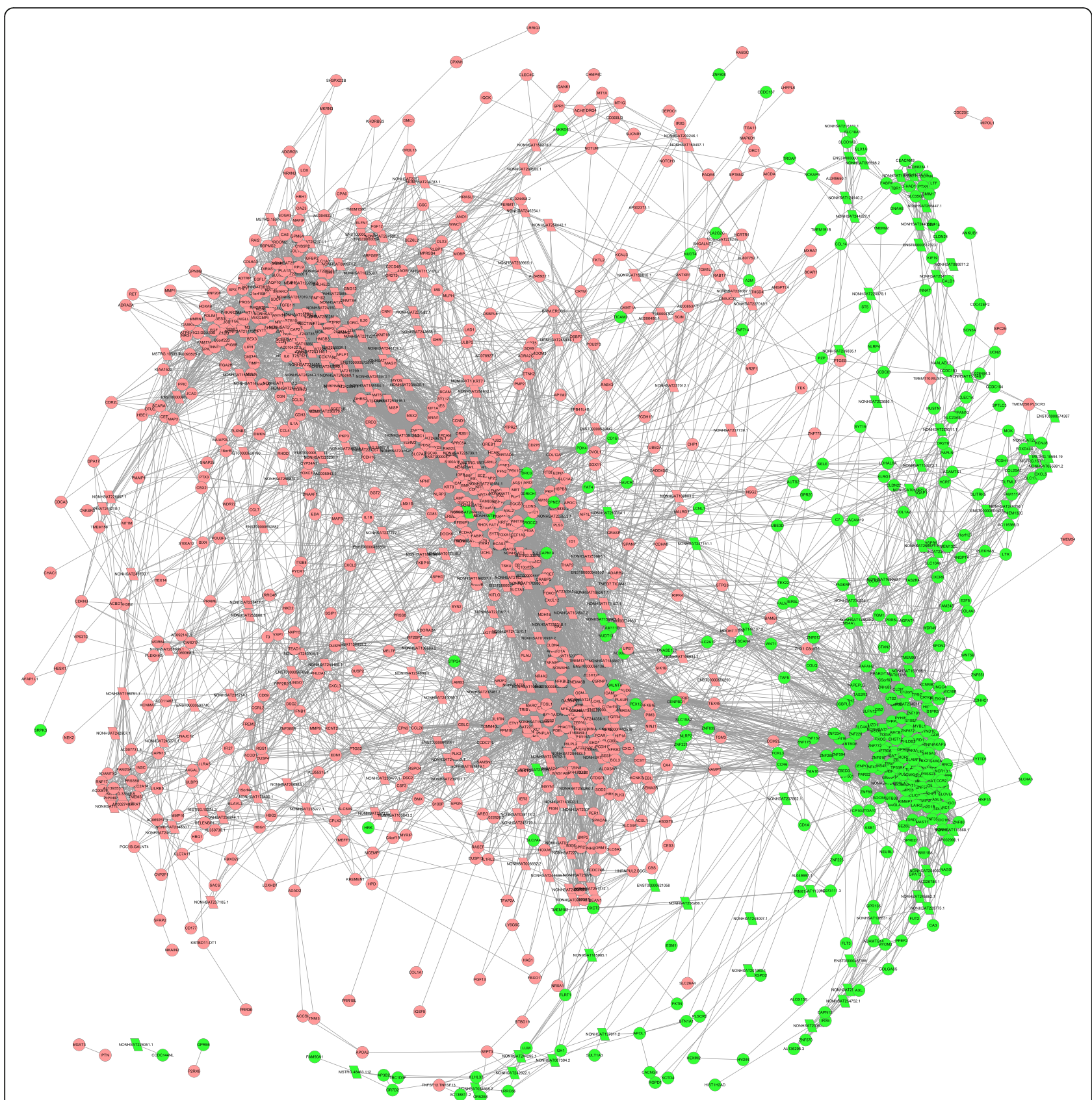

Fig. 3 AS-related IncRNA-mRNA interaction network. An interaction network was constructed incorporating differentially expressed genes, with up- and down-regulated genes being marked in red and green, respectively

rheumatic diseases such as Rheumatoid Arthritis (RA), systemic lupus erythematosus (SLE), and multiple sclerosis (MS). These other rheumatic diseases should be considered in similar future studies so as to strengthen the argument supporting that differentially expressed lncRNAs are useful diagnostic biomarkers for AS.

\section{Conclusion}

In summary, we utilized an RNA-seq approach to characterize AS-related lncRNA expression profiles in patient PBMCs. The identified differentially expressed lncRNAs may offer new insight into the molecular etiology of this complex and debilitating disease. However, future mechanistic analyses will be required 

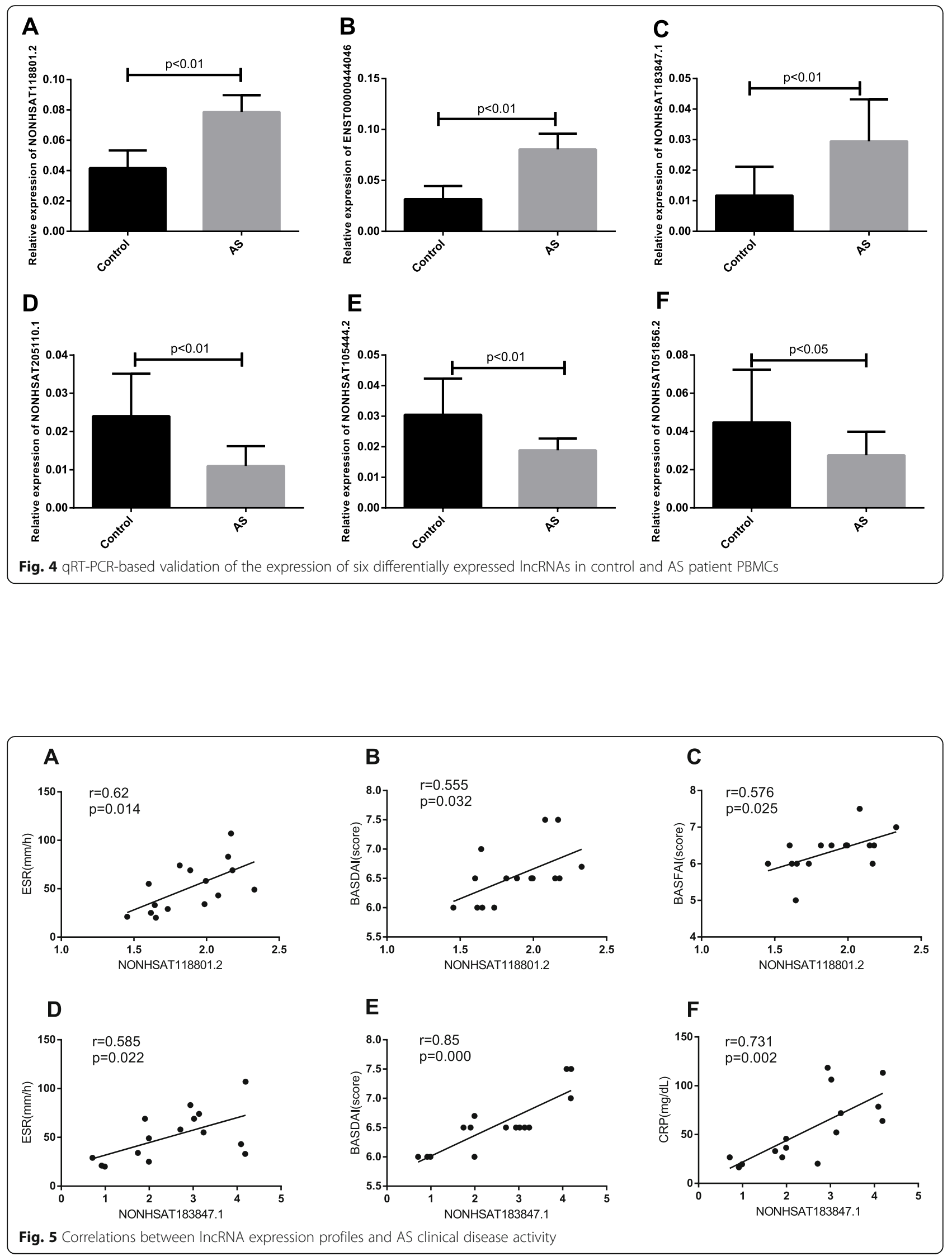
in order to validate our findings and to fully explore the clinical and therapeutic relevance of these lncRNAs in this disease.

\section{Abbreviations}

AS: Ankylosing spondylitis; IncRNAs: Long non-coding RNAs;; RNA-seq: Highthroughput RNA sequencing; PBMC: Peripheral blood mononuclear cell; qRTPCR: Quantitative real-time polymerase chain reaction

\section{Acknowledgements}

Not applicable.

\section{Authors' contributions}

$J L$ made substantial contributions to the conception and design of the study. DH, YYF, YL and YZ performed the experiments. DH and BXB contributed to data acquisition, and wrote the main manuscript. JL and LW revised the manuscript critically for important intellectual content. All authors agreed to be accountable for all aspects of the work in ensuring that questions related to the accuracy or integrity of the work are appropriately investigated and resolved. All authors read and approved the final manuscript.

\section{Funding}

This project was supported by National key research and development program of the ministry of science and technology (2018YFC1705204). Anhui provincial laboratory of internal medicine application and development of modern Chinese medicine (2016080503B041), Construction project of Jian Liu studio of TCM in Anhui province (secretary of development of traditional Chinese medicine [2018] no. 11). Fundings will be used to pay for costs associated with RNA-seq analysis. The funding body will not assist with study design, data interpretation and manuscript writing.

\section{Availability of data and materials}

The datasets generated and/or analysed during the current study are available in National Genomics Data Center (Nucleic Acids Res 2021), China National Center for Bioinformation/Beijing Institute of Genomics, Chinese Academy of Sciences, under accession number PRCA004516 that are publicly accessible at https://bigd.big.ac.cn/gsa.

\section{Declarations}

\section{Consent to publication}

Not applicable.

\section{Ethics approval and consent to participate}

This study was approved and stricktly followed by the Medical Ethics Committee of the First Affiliated Hospital of Anhui University of Chinese Medicine (2015AH-20), A prior written informed consent was obtained from all the subjects to participate in the study, all methods were performed in accordance with the relevant guidelines and regulations.

\section{Competing interests}

The authors declare that they have no competing interests.

Received: 22 July 2020 Accepted: 25 February 2021

\section{Published online: 12 March 2021}

\section{References}

1. Liu G, Ma Y, Yang Q, Deng S. Modulation of inflammatory response and gut microbiota in ankylosing spondylitis mouse model by bioactive peptide IQW. J Appl Microbiol. 2020;128(6):1669-77.

2. Ghasemi-Rad M, Attaya H, Lesha E, Vegh A, Maleki-Miandoab T, Nosair E, Sepehrvand N, Davarian A, Rajebi H, Pakniat A, et al. Ankylosing spondylitis: a state of the art factual backbone. World J Radiol. 2015;7(9):236-52.

3. Ermann J. Pathogenesis of axial Spondyloarthritis - sources and current state of knowledge. Rheum Dis Clin N Am. 2020;46(2):193-206.

4. Sveaas SH, Berg IJ, Provan SA, Semb AG, Olsen IC, Ueland T, Aukrust P, Vøllestad N, Hagen KB, Kvien TK, et al. Circulating levels of inflammatory cytokines and cytokine receptors in patients with ankylosing spondylitis: a cross-sectional comparative study. Scand J Rheumatol. 2015;44(2):118-24.

5. Rashid T, Ebringer A. Ankylosing spondylitis is linked to Klebsiella - the evidence. Clin Rheumatol. 2006;26(6):858-64.
6. Zeng L, Lindstrom MJ, Smith JA. Ankylosing spondylitis macrophage production of higher levels of interleukin-23 in response to lipopolysaccharide without induction of a significant unfolded protein response. Arthritis Rheum. 2011;63(12):3807-17.

7. Mercer TR, Dinger ME, Mattick JS. Long non-coding RNAs: insights into functions. Nat Rev Genet. 2009;10(3):155-9.

8. Aune TM, Crooke PS 3rd, Patrick AE, Tossberg JT, Olsen NJ, Spurlock CF 3rd. Expression of long non-coding RNAs in autoimmunity and linkage to enhancer function and autoimmune disease risk genetic variants. J Autoimmun. 2017:81:99-109.

9. Li J, Li Z, Leng K, Xu Y, Ji D, Huang L, Cui Y, Jiang X. ZEB1-AS1: A crucial cancer-related long non-coding RNA. Cell Prolif. 2018;51(1):e12423.

10. Huang Z, Zhou J-K, Peng Y, He W, Huang C. The role of long noncoding RNAs in hepatocellular carcinoma. Mol Cancer. 2020;19(1):77.

11. Xu H, Chen W, Zheng F, Tang D, Liu D, Wang G, Xu Y, Yin L, Zhang X, Dai Y. Reconstruction and analysis of the aberrant IncRNA-miRNA-mRNA network in systemic lupus erythematosus. Lupus. 2020;29(4):398-406.

12. Cao HY, Li D, Wang YP, Lu HX, Sun J, Li HB. Clinical significance of reduced expression of IncRNA TUG1 in the peripheral blood of systemic lupus erythematosus patients. Int J Rheum Dis. 2020;23(3):428-34.

13. Li J, Wu G-C, Zhang T-P, Yang X-K, Chen S-S, Li L-J, Xu S-Z, Lv T-T, Leng R-X, Pan $\mathrm{H}-\mathrm{F}$, et al. Association of long noncoding RNAs expression levels and their gene polymorphisms with systemic lupus erythematosus. Sci Rep. 2017;7(1):15119.

14. Ghaiad HR, Elmazny AN, Nooh MM, El-Sawalhi MM, Shaheen AA. Long noncoding RNAs APOA1-AS, IFNG-AS1, RMRP and their related biomolecules in Egyptian patients with relapsing-remitting multiple sclerosis: relation to disease activity and patient disability. J Adv Res. 2019;21:141-50.

15. Moradi A, Naiini MR, Yazdanpanahi N, Tabatabaeian H, Nabatchian F, Baghi M, Azadeh M, Ghaedi K. Evaluation of The Expression Levels of Three Long Non-Coding RNAs in Multiple Sclerosis. Cell J (Yakhteh). 2020;22:2.

16. Yan S, Wang P, Wang J, Yang J, Lu H, Jin C, Cheng M, Xu D. Long non-coding RNA HIX003209 promotes inflammation by sponging miR-6089 via TLR4/NF-KB signaling pathway in rheumatoid arthritis. Front Immunol. 2019;10:2218.

17. Yuan M, Wang S, Yu L, Qu B, Xu L, Liu L, Sun H, Li C, Shi Y, Liu H. Long noncoding RNA profiling revealed differentially expressed IncRNAs associated with disease activity in PBMCs from patients with rheumatoid arthritis. PLoS One. 2017;12(11):e0186795.

18. Zhou Q, Yu Q, Gong Y, Liu Z, Xu H, Wang Y, Shi Y. Construction of a IncRNA-miRNA-mRNA network to determine the regulatory roles of IncRNAs in psoriasis. Exp Ther Med. 2019;18(5):4011-21.

19. Linden SVD, Valkenburg HA, Cats A. Evaluation of diagnostic criteria for ankylosing spondylitis. Arthritis Rheum. 1984;27(4):361-8.

20. Garrett S, Jenkinson T, Kennedy LG, Whitelock H, Gaisford P, Calin A. A new approach to defining disease status in ankylosing spondylitis: the Bath ankylosing spondylitis disease activity index. J Rheumatol. 1994;21 (12):2286-91.

21. Calin A, Garrett S, Whitelock H, Kennedy LG, O'Hea J, Mallorie P, Jenkinson T. A new approach to defining functional ability in ankylosing spondylitis: the development of the Bath ankylosing spondylitis functional index. J Rheumatol. 1994;21(12):2281-5.

22. Ruiz-Orera J, Messeguer X, Subirana JA, Alba MM. Long non-coding RNAs as a source of new peptides. Elife. 2014;3:e03523.

23. Xu Z, Zhou X, Hao L, Chen Q, Chen G. Identification of the key genes and long non-coding RNAs in ankylosing spondylitis using RNA sequencing. Int J Mol Med. 2019;43:1179-92.

24. Zhang C, Wang C, Jia Z, Tong W, Liu D, He C, Huang X, Xu W. Differentially expressed mRNAs, IncRNAs, and miRNAs with associated co-expression and ceRNA networks in ankylosing spondylitis. Oncotarget. 2017:8:113543-57.

25. Li X, Chai W, Zhang G, Ni M, Chen J, Dong J, Zhou Y, Hao L, Bai Y, Wang Y. Down-regulation of IncRNA-AK001085 and its influences on the diagnosis of ankylosing spondylitis. Med Sci Monit. 2017;23:11-6.

26. Qin X, Jiang T, Liu S, Tan J, Wu H, Zheng L, Zhao J. Effect of metformin on ossification and inflammation of fibroblasts in ankylosing spondylitis: an in vitro study. J Cell Biochem. 2017;119(1):1074-82.

\section{Publisher's Note}

Springer Nature remains neutral with regard to jurisdictional claims in published maps and institutional affiliations. 